\title{
A NEW UPPER BOUND FOR 1324-AVOIDING PERMUTATIONS
}

\author{
MIKLÓS BÓNA
}

\begin{abstract}
We prove that the number of 1324-avoiding permutations of length $n$ is less than $(7+4 \sqrt{3})^{n}$.
\end{abstract}

\section{INTRODUCTION}

1.1. Definitions and Open Questions. The theory of pattern avoiding permutations has seen tremendous progress during the last two decades. The key definition is the following. Let $k \leq n$, let $p=p_{1} p_{2} \cdots p_{n}$ be a permutation of length $n$, and let $q=q_{1} q_{2} \cdots q_{k}$ be a permutation of length $k$. We say that $p$ avoids $q$ if there are no $k$ indices $i_{1}<i_{2}<\cdots<i_{k}$ so that for all $a$ and $b$, the inequality $p_{i_{a}}<p_{i_{b}}$ holds if and only if the inequality $q_{a}<q_{b}$ holds. For instance, $p=2537164$ avoids $q=1234$ because $p$ does not contain an increasing subsequence of length four. See [3] for an overview of the main results on pattern avoiding permutations.

The shortest pattern for which even some of the most basic questions are open is $q=1324$, a pattern that has been studied for at least 17 years. For instance, there is no known exact formula for the number $S_{n}(1324)$ of permutations of length $n$ (or, in what follows, $n$-permutations) avoiding 1324. Even the value of $L(1324)=\lim _{n \rightarrow \infty} \sqrt[n]{S_{n}(1324)}$ is unknown, though the limit is known to exist 2 .

The best known upper bound for the numbers $S_{n}(1324)$ was given in 2011 by Claesson, Jelinek and Steingrímsson [5] who proved that for all positive integers $n$, the inequality $S_{n}(1324)<16^{n}$ holds. The best known lower bound, $S_{n}(1324) \geq 9.42^{n}$, was given by five authors in 1 in 2005 .

In this paper, we prove the inequality $S_{n}(1324)<(7+4 \sqrt{3})^{n}$. The proof introduces a refined version of a decomposition of 1324-avoiding permutations given in [5], encodes such permutations by two words over a 4-element alphabet, and then enumerates those words.

1.2. Preliminaries. In this section, we present a few simple facts that are well-known among researchers working in the area that will be necessary in order to understand some of our proofs in the subsequent sections. Readers familiar with the area may skip this section. Proofs that are not given here can be found in [3].

Theorem 1.1. Let $q$ be any pattern of length three. Then $S_{n}(q)=C_{n}=$ $\left(\begin{array}{c}2 n \\ n\end{array}\right) /(n+1)$, the nth Catalan number. In particular, $S_{n}(q)<4^{n}$. 
An entry of a permutation is called a left-to-right minimum if it is smaller than all entries on its left. Right-to-left maxima are defined analogously. For instance, in $p=351624$, the left-to-right minima are 3 and 1, while the right-to-left maxima are 6 and 4. A 132-avoiding permutation is completely determined by the set of its left-to-right minima, and the set of indices that belong to entries that are left-to-right minima. Indeed, left-to-right minima must always be in decreasing order. Furthermore, once the set and position of the left-to-right minima are given, the order of elements that are not leftto-right minima is uniquely determined. To see this, fill the positions that belong to entries that are not left-to-right minima one by one, going left to right. In each step, the smallest remaining entry that is larger than the closest left-to-right minimum $m$ on the right of the position at hand must be placed. If we do not follow this procedure and place the entry $y$ instead of the smaller entry $x$, then the 132-pattern myx is formed. For example, to find the unique permutation of length 6 whose left-to-right minima are the entries 1, 3, and 4, and that has left-to-right minimia in the first, second and fifth position, write the left-to-right minima in the specified positions in decreasing order, to get $43 * * 1 *$, where the $*$ denote positions that are still empty. Then fill the empty slots with the remaining entries, always placing the smallest entry that is larger than the closest left-to-right minimum on the left. In this case, that means first placing 5 , then 6 , then 2 , to get 435612 .

In an analogous way, each 213-avoiding permutation is determined by the set of its right-to-left maxima, and the set of indices that belong to right-to-left maxima.

In preparation to our main results, we reformulate the facts discussed in the last paragraphs. Permutations $p=p_{1} p_{2} \cdots p_{n}$ of length $n$ that avoid 132 can be injectively encoded by ordered pairs of words $(u(p), v(p))$ of length $n$ defined as follows. The $i$ th letter of $u(p)$ is 0 if $p_{i}$ is a left-to-right minimum in $p$, and 1 otherwise. The $i$ th letter of $v(p)$ is 0 if the entry $i$ is a left-to-right minimum in $p$, and 1 otherwise. The encoding of 213 -avoiding permutations is analogous.

\section{Coloring Entries}

The starting point of our proof is the following decomposition of 1324avoiding permutations, given in 5 .

Let $p=p_{1} p_{2} \cdots p_{n}$ be a 1324-avoiding permutation, and let us color each entry of $p$ red or blue as we move from left to right, according the following rules.

(1) If coloring $p_{i}$ red would create a 132-pattern with all red entries, then color $p_{i}$ blue, and

(2) if there already is a blue entry smaller than $p_{i}$, then color $p_{i}$ blue;

(3) otherwise color $p_{i}$ red. 
It is then proved in [5] that the red entries form a 132-avoiding permutation and the blue entries form a 213 -avoiding permutation. From this, it is not difficult to prove that the number of 1324 -avoiding $n$-permutations is less than $16^{n}$. Indeed, there are at most $2^{n}$ possibilities for the set of the red entries (the blue entries being the remaining entries), and there are at most $2^{n}$ possibilities for the positions in which red entries are placed (the blue entries then must be placed in the remaining positions). Once the set

and positions of the $k$ red entries are known, there are $C_{k}<4^{k}$ possibilities for their permutation, just as there are $C_{n-k}<4^{n-k}$ possibilities for the permutation of the blue entries, completing the proof of the inequality $S_{n}(1324)<16^{n}$.

\section{REFINING THE COLORING}

In this section, we improve the upper bound on $S_{n}(1324)$ by using a more refined decomposition of 1324-avoiding permutations, which enables us to carry out a more careful counting argument. Let us color each entry of the 1324-avoiding permutation $p=p_{1} p_{2} \cdots p_{n}$ red or blue as in Section 2 , Furthermore, let us mark each entry of $p$ with one of the letters $A, B, C$, or $D$ as follows.

(1) Mark each red entry that is a left-to-right minium in the partial permutation of red entries by $A$,

(2) mark each red entry that is not a left-to-right minimum in the partial permutation of red entries by $B$,

(3) mark each blue entry that is not a right-to-left maximum in the partial permutation of blue entries by $C$, and

(4) mark each blue entry that is a right-to-left maximum in the partial permutation of blue entries by $D$.

Call entries marked by the letter $X$ entries of type $X$. Let $w(p)$ be the $n$-letter word over the alphabet $\{A, B, C, D\}$ defined above. In other words, the $i$ th letter of $w(p)$ is the type of $p_{i}$ in $p$. Let $z(p)$ be the $n$-letter word over the alphabet $\{A, B, C, D\}$ whose $i$ th letter is the type of the entry $i$ in $p$.

Example 3.1. Let $p=3612745$. Then the subsequence of red entries of $p$ is 36127 , the subsequence of blue entries of $p$ is 45 , so $w(p)=A B A B B C D$, while $z(p)=A B A C D B B$.

The following lemma shows a property of $w(p)$ that will enable us to improve the upper bound on $S_{n}(1324)$. Let us say that a word $w$ has a $C B$-factor if somewhere in $w$, a letter $C$ is immediately followed by a letter $B$.

Lemma 3.2. If $p$ is 1324-avoiding, then $w(p)$ has no $C B$-factor.

Proof. Let us assume that $C_{1}$ is the $i$ th letter of $w(p)$, and $B_{1}$ is the $(i+1)$ st letter of $w(p)$. That means that $p_{i}>p_{i+1}$, otherwise the fact that $p_{i}$ is 
blue would force $p_{i+1}$ to be blue. Furthermore, since $p_{i}$ is not a right-toleft maximum, there is an entry $d$ on the right of $p_{i}$ (and on the right of $\left.p_{i+1}\right)$ so that $p_{i}<d$. Similarly, since $p_{i+1}$ is not a left-to-right minimum, there is an entry $a$ on its left so that $a<p_{i+1}$. However, then $a p_{i} p_{i+1} d$ is a 1324-pattern, which is a contradiction.

Lemma 3.3. If $p$ is 1324-avoiding, then there is no entry $i$ in $p$ so that $i$ is of type $C$ and $i+1$ is of type $B$.

Proof. Analogous to the proof of lemma 3.2, If such a pair existed, $i$ would have to be on the right of $i+1$, since $i$ is blue and $i+1$ is red. As $i$ is not a right-to-left maximum, there would be a larger entry $d$ on its right. As $i+1$ is not a left-to-right minimum, there would be a smaller entry $a$ on its left. However, then $a(i+1) i d$ would be a 1324-pattern.

Lemma 3.4. Let $h_{n}$ be the number of words of length $n$ that consist of letters $A, B, C$ and $D$ that have no $C B$-factors. Then we have

$$
H(x)=\sum_{n \geq 0} h_{n} x^{n}=\frac{1}{1-4 x+x^{2}} .
$$

This implies

$$
h_{n}=\frac{3+2 \sqrt{3}}{6} \cdot(2+\sqrt{3})^{n}+\frac{3-2 \sqrt{3}}{6} \cdot(2-\sqrt{3})^{n} .
$$

Proof. We claim that if $n \geq 2$, then $h_{n}=4 h_{n-1}-h_{n-2}$. Indeed, take any of the $h_{n-1}$ words of length $n-1$ that have the given property. Affix any of the four letters of the alphabet to the end of each such word. The result is a word counted by $h_{n}$, except in the $h_{n-2}$ cases in which the last two letters are $C$ and $B$, in that order.

Together with the initial conditions $h_{0}=1$ and $h_{1}=4$, this leads to the functional equation

$$
H(x)-4 x-1=4 x(H(x)-1)-x^{2} H(x) .
$$

Expressing $H(x)$, we obtain

$$
H(x)=\frac{1}{1-4 x+x^{2}}
$$

as claimed. It is now routine to find the exact formula for $h_{n}$ using partial fractions.

The following, simple but crucial lemma tells us that the ordered pair $(w(p), z(p))$ completely determines the 1324-avoiding permutation $p$. Readers who prefer may consult Section 1.2 first for some background on this argument.

Lemma 3.5. Let $A v_{n}(1324)$ be the set of all 1324-avoiding n-permutations. Then the map $f: A v_{n}(1324) \rightarrow H_{n} \times H_{n}$, given by $f(p)=(w(p), z(p))$ is injective. 
Proof. Let $H_{n}$ be the set of all words of length $n$ over the alphabet $\{A, B, C, D\}$ in which a letter $C$ is never immediately followed by a letter $B$. Then $\left|H_{n}\right|=h_{n}$. Let $(w, z) \in H_{n}$, and let us assume that $f(p)=(w, z)$, that is, that $w(p)=w$, and $z(p)=z$ for some $p \in A v_{n}(1324)$.

Then $w$ tells us for which indices $i$ the entry $p_{i}$ will be of type $A$, namely for the indices $i$ for which the $i$ th letter of $w$ is $A$. Similary, $w$ tells us the indices $j$ for which the entry $p_{i}$ is of type $B$, type $C$, or type $D$.

After this, we can use $z$ to figure out which entries of $p$ are of type $A$, type $B$, type $C$ or type $D$.

There remains to show that this information completely determines $p$, that is, that there is at most one permutation that avoids 1324 and satisfies all the type requirements imposed by $w$ and $z$.

In order to see this, note that entries of type $A$ must be in decreasing order in their positions. Entries of type $D$ must be in decreasing order in their positions. Once these entries are placed, entries of type $B$ must be placed in their positions from left to right, so that in each step, the smallest available entry is placed that is larger than the closest entry of type $A$ on the left. (Otherwise a red 132-pattern is formed.) Similarly, the entries of type $D$ must be placed in their positions from the right, so that in each step, the largest available entry is placed that is smaller than the closest right-to-left maximum on the right. (Otherwise a blue 213-pattern is formed.)

Corollary 3.6. For all positive integers $n$, the inequality

$$
S_{n}(1324)<h_{n-1}^{2}
$$

holds.

Proof. The fact that $S_{n}(1324)<h_{n}^{2}$ is immediate from the injective property of $f$ that we have just proved in Lemma3.5. In order to complete the proof of this Corollary, note that the image of $f$ consists of ordered pairs $(w(p), z(p))$ in which both $w(p)$ and $z(p)$ starts with an $A$, since both $p_{1}$ and 1 are always red, and left-to-right minima within the string of red entries (and even in all of $p$ ).

\section{Acknowledgment}

I am grateful to Vincent Vatter for some valuable remarks streamlining the proofs of two of my lemmas.

\section{REFERENCES}

[1] M. H. Albert, M. Elder, A. Rechnitzer, P. Westcott, M. Zabrocki, On the StanleyWilf limit of 4231-avoiding permutations and a conjecture of Arratia, Adv. in Appl. Math. 36 (2006), no. 2, 96-105.

[2] R. Arratia, On the Stanley-Wilf conjecture for the number of permutations avoiding a given pattern, Electronic J. Combin., 6 (1999), no. 1, N1.

[3] M. Bóna, Combinatorics of Permutations, second edition, CRC Press - Chapman Hall, 2012.

[4] M. Bóna, Records in Stanley-Wilf limits, European J. Combin., 28 (2007), no. 1, 75 -V85. 
[5] A. Claesson, V. Jelinek, E. Steingrímsson, Upper bounds for the Stanley-Wilf limit of 1324 and other layered patterns. Preprint, arXiv:1111.5736v1, November 24, 2011, submitted to Elsevier.

M. Bóna, Department of Mathematics, University of Florida, 358 Little Hall, PO Box 118105, Gainesville, FL 32611-8105 (USA) 